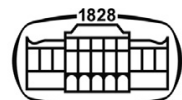

AKADÉMIAI KIADÓ

Journal of Behavioral Addictions

9 (2020) 2, 312-324

DOl:

10.1556/2006.2020.00029

(c) 2020 The Author(s)

\section{FULL-LENGTH REPORT}

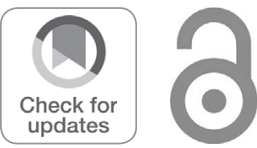

\title{
Inhibitory neuromodulation of the putamen to the prefrontal cortex in Internet gaming disorder: How addiction impairs executive control
}

\author{
MIN WANG ${ }^{1}, \mathrm{HAOHAO}$ DONG $^{2}, \mathrm{HUI} \mathrm{ZHENG}^{3}$, XIAOXIA DU ${ }^{4}$ \\ and GUANG-HENG DONG ${ }^{1,5 *}$ (1)
}

${ }^{1}$ Center for Cognition and Brain Disorders, the Affiliated Hospital of Hangzhou Normal University, Hangzhou, PR China

${ }^{2}$ Department of Psychology, Zhejiang Normal University, Jinhua, PR China

${ }^{3}$ Shanghai Key Laboratory of Psychotic Disorders, Shanghai Mental Health Center, Shanghai Jiaotong University School of Medicine, Shanghai, PR China

${ }^{4}$ Department of Physics, Shanghai Key Laboratory of Magnetic Resonance, East China Normal University, Shanghai, PR China

${ }^{5}$ Zhejiang Key Laboratory for Research in Assessment of Cognitive Impairments, Hangzhou, Zhejiang Province, PR China

Received: September 12, 2019 • Revised manuscript received: February 21, 2020; March 18, 2020 • Accepted: April 4, 2020 • Published online: July 9, 2020

\begin{abstract}
Background and aims: Individuals with addictive disorders are usually characterized by impaired executive control, persistent craving and excessive reward-seeking. However, it is unclear whether there is a deviation in the connection pattern among the neural systems implicated in these problem behaviors. Methods: One hundred thirty-six online gaming players were recruited in the current study (68 Internet gaming disorder (IGD) subjects and 68 recreational game users (RGUs) who served as controls matched on age, sex, years of education, and years of gaming). Dynamic interactions among the reward system (striatum), control system (prefrontal cortex), and the interoceptive awareness system (insula) were calculated and compared when subjects were facing gaming cues. Results: The results revealed that RGUs showed a significant positive correlation in the putamen-middle frontal gyrus (MFG)-insula neural pathway when facing gaming cues, which was missing in the IGD subjects. Additionally, dynamic causal modeling (DCM) analysis revealed that the MFG region was more inhibited by the putamen in the IGD subjects relative to the RGUs. Conclusions: These findings suggest that the inhibitory neuromodulation of the putamen to the prefrontal cortex in IGD individuals undermines the balance among the tripartite systems. Our findings provide novel neurobiological evidence for understanding the internal connection bias of the addicted individual's neural system and how the addictive disorder impairs executive control; consequently, the pathway from the striatum to the prefrontal cortex may serve as a potential biomarker to predict the risk of developing an addiction.
\end{abstract}

\section{KEYWORDS}

addiction, reward, executive control, internet gaming disorder, dynamic causal modeling

\section{INTRODUCTION}

*Corresponding author.

E-mail: dongguangheng@hznu.edu.cn

Several behaviors including problematic game, gamble, sex, and shopping, besides psychoactive substance ingestion, seek the short-term reward that may lead to persistent behavior despite adverse consequences, i.e., diminished executive control. Diminished control is a central conceptualization of substance dependence. This phenomenological similarity has given rise to the concept of behavioral addiction (Grant, Potenza, Weinstein, \& Gorelick, 2010), i.e., non- 
substance addictions with a behavioral focus other than substance ingestion. It shares many clinical characteristics and similar neural mechanisms with substance dependence, involving the disruption of the limbic circuitry (Koob \& Le Moal, 2001) and prefrontal cortex network (Feil et al., 2010). Currently, these addictive disorders expanding from substance to non-substance have become a substantial public health concern.

Individuals with addictive disorders, including behavioral addiction and even certain addictive-like behaviors (e.g., obesity), are often characterized by impaired executive control, persistent craving and excessive reward-seeking (Bari \& Robbins, 2013; Zheng et al., 2019). Three systems in the brain are thought to be involved (Noel, Brevers, \& Bechara, 2013): 1) the reward system, which involves the orbitofrontal cortex (OFC)-striatum-amygdala pathway, and drives reward-seeking, 2) the control system, which involves the prefrontal cortex and executes cognitive control, and 3) the interoceptive awareness system, which involves the insula and responds to craving states. Neurobiological studies have provided clear evidence implicating changed activity in the above regions following long-term drug intake or problem behaviors. For example, individuals with drug dependence show hypoactivation of the prefrontal region during inhibition, including the dorsolateral prefrontal cortex, middle frontal gyrus (MFG), and anterior cingulate cortex (Luijten et al., 2014; Spechler et al., 2016). The hyperactivation of reward regions involving the striatum also plays a crucial role in the course of addiction (Kringelbach, O’Doherty, Rolls, \& Andrews, 2003). Similarly, abnormalities in prefrontal and striatum region activity has been also observed in behavioral addiction (e.g., Internet gaming disorder, IGD) (Dong, Wang, Du, \& Potenza, 2017). In addition, this reward-related activity also extends into the insula, OFC and thalamus in response to craving triggered by reward cues such as drugs, money, gaming and food images (Huckins et al., 2019; Sescousse, Caldu, Segura, \& Dreher, 2013; Somerville, Hare, \& Casey, 2011; Yarkoni, Poldrack, Nichols, Van Essen, \& Wager, 2011).

Although these three systems are generally considered to be independent structures that contributed to addictive behaviors, there is evidence that they are functionally related, perhaps interacting with each other to induce inappropriate or maladaptive behaviors. For instance, a lower functional connectivity (FC) between the dorsolateral prefrontal cortex (DLPFC) and striatum was observed in alcohol addicts than in healthy controls during monetary reward (Becker, Kirsch, Gerchen, Kiefer, \& Kirsch, 2017). In spontaneous activities, substance abusers have shown a lower resting-state FC between nucleus accumbens and frontal cortical regions than non-substance abuse subjects (Motzkin, Baskin-Sommers, Newman, Kiehl, \& Koenigs, 2014). Another study on IGD demonstrated that the coupling between decreased DLPFC activity and increased insula activity might underlie continuous addictive behaviors (Dong, Liu, Zheng, Du, \& Potenza, 2019a). Additionally, increased connectivity between the insula and the DLPFC was observed in individuals addicted to nicotine after treatment (Li et al., 2019). These findings suggests that dysregulation of the neural response in addicted individuals might be related to this relative imbalance among the tripartite systems. If this is the case, this imbalance might serve as a critical marker for predicting the risk of addiction and provide a potential target for subsequent treatment.

The changes of information flow among the tripartite system in addicted individuals, which might be prospective risk factors contributing to the intersystem imbalance, remain unclear. Preliminary prospective studies show that neural correlations between the executive control and reward systems in the normal population are negatively associated (Weafer, Crane, Gorka, Phan, \& de Wit, 2019). Moreover, another study shows that the insula could further sensitize the reward system and inhibit the control system, when facing reward stimuli, suggesting a potential causal relationship to predict addiction (He et al., 2019). It should be noted that although these findings are valuable in understanding the imbalance among the tripartite systems in addicts, they could not conclude that the above associations among the systems could be equally characterized in addicted individuals. Put simply, it is difficult to directly map the information flow interactions among tripartite systems in addicted individuals only using the current evidence.

IGD is a behavioral addiction that specifically refers to excessive indulge in online games and results in a series of cognitive deficits (Kuss, Pontes, \& Griffiths, 2018). IGD is usually regarded as one sub-type of Internet addiction. Comparing with other types of online behaviors of Internet addiction, online games appear as particularly strong risk factors for addiction (Kuss, 2013; Kuss, Griffiths, \& Binder, 2013; Kuss, Griffiths, \& Pontes, 2017). In 2013, section 3 of the DSM-5 included IGD as a condition for further study and proposed a specialized diagnostic criterion (DSM-5, https://www.psychiatry.org/psychiatrists/practice/dsm),

which have promoted further systematization of these imaging studies specific to excessive game behaviors. In addition, more and more neurological evidence has revealed the similarities between substance dependence and IGD, including functional and structural changes located on the prefrontal and limbic lobe (Kuss \& Griffiths, 2012; Petry, Zajac, \& Ginley, 2018). In the current study, we selected the IGD populations to explore the neural coupling among the tripartite systems associated with addiction.

We first computed the correlations among three neural systems in IGD during different cue reactivity to explore stable inter-system association patterns; meanwhile, recreational game users (RGUs) were used as a control group. The dual process theory of addiction suggests that when facing reward stimuli, addicted individuals have a hyperactive reward system that drives the individual to further rewardseeking behavior. On the other hand, a hypoactive control system might impair cognitive control and the capbility to optimally weigh short term benefits against long term losses underlying addictive behavior. In addition, the interoceptive awareness system might convert the subjectively experienced craving signal into a neural impulse to further promote the occurrence of problem behaviors (Bechara, 2005; He et al., 2019; Kelley \& Berridge, 2002; Volkow \& Baler, 2015; 
Volkow \& Wise, 2005). According to this theory, we hypothesized that these two groups have different inter-system association patterns and that the relative imbalance of the patterns makes it difficult for IGD to suppress self-craving and impulsive behaviors compared with RGUs.

Another challenge was how to quantify the risk factors that might contribute to intersystem imbalances in IGD. Here, we examined the neural dynamics among three neural systems in individuals with IGD when they were exposed to gaming cues. Specifically, we used dynamic causal modeling (DCM) to separately construct effective connectivity patterns among three systems for individuals with IGD and RGUs during gaming cue reactivity and characterize possible risk factors by comparing differences between the groups. Preliminary prospective studies have demonstrated negative neural correlations between control and reward systems (Becker et al., 2017; Weafer et al., 2019). Additionally, a cognitive-behavioral model of IGD proposes that motivational drives associated with reward-seeking behaviors contribute crucially to IGD and that diminished inhibitory control over this reward seeking contributes to decisionmaking that leads to persistent addictive behaviors (Dong \& Potenza, 2014). In light of this, we further hypothesized that the anomalous communications between the reward and control systems in IGD might be a potential risk factor contributing to an imbalance among the tripartite systems.

\section{METHODS}

\section{Participants}

A total of 143 online gaming players from universities in Shanghai China participated in the current study, recruited through public posters and online forms. Seven males, including 6 RGU and 1 IGD subject, were excluded owing to nicotine dependence, resulting in a final sample of one hundred thirty-six subjects (68 IGD subjects, 68 RGUs). For each participant, structured psychiatric interviews (MINI) (Sheehan et al., 1998) was conducted by the trained members of the research staff for excluding individuals with a history of other psychiatric disorders (e.g., anxiety, depression, and bipolar disorders), or substance abuse. The Internet addiction test (IAT) by Young (Chang \& Law, 2008) and the nine criteria provided in the DSM-5 (Petry et al., 2014) was used to characterize IGD. Subjects that simultaneously satisfied more than 5 of the DSM-5 criteria and an IAT score greater than 50 were labeled as having IGD, and RGUs met less than 5 of the DSM-5 criteria or scored less than 50 on Young's IAT. The demographic factors of the participants are detailed in Table 1 , of which age, sex $\left(\chi^{2}=2.982, P=0.084\right)$ and self-reported craving were used as covariates for subsequent statistical analysis.

\section{PROCEDURE}

\section{Cue-craving task}

The cue craving task used in the current study was described in detail elsewhere (Dong, Liu, et al., 2019a; Dong, Wang, et al., 2019b) and described briefly below (see Fig. 1). In brief, the screen first presented a fixed cross that lasted for $500 \mathrm{~ms}$. Next, screen would present the cues that were divided into two categories: gaming cues and neutral cues. In the gaming cues, a player was shown sitting in front of a computer and playing an online game, with half of the cues showing the player's profile in such a way that eliminated facial emotional interference and the other half showing only the player's hands. The neutral cues were matched to the gaming cues, replacing the game with a document. The subjects needed to respond as to whether a profile or hands appeared when showing the cues. The duration of the cues was determined by the button reaction of the subjects, while the cues presentation time was up to a maximum of 3,000 ms when the subjects did not respond. A blank screen was presented after the response and lasted for (3,000 - response time) ms. In the subsequent evaluation stage, participants assessed their gaming craving through a 5-point scale (5 means the highest degree of

Table 1. Demographic information and group differences in current study

\begin{tabular}{|c|c|c|c|c|}
\hline & $\begin{array}{c}\text { IGD (68) } \\
\text { Male }=33, \text { female }=35\end{array}$ & $\begin{array}{c}\text { RGU (68) } \\
\text { male }=43, \text { female }=25\end{array}$ & $t$ & $P$ \\
\hline Age $($ mean $\pm S D)$ & $20.912 \pm 2.012$ & $21.615 \pm 2.218$ & -1.772 & 0.079 \\
\hline IAT score $($ mean $\pm \mathrm{SD})$ & $64.000 \pm 10.108$ & $37.338 \pm 8.038$ & 17.024 & 0.000 \\
\hline DSM-5 score $($ mean \pm SD) & $5.794 \pm 1.073$ & $2.367 \pm 1.268$ & 17.007 & 0.000 \\
\hline Gaming playing hours index $($ mean $\pm S D)$ & $25.205 \pm 3.818$ & $14.955 \pm 5.862$ & 12.080 & 0.000 \\
\hline Educations (years) (mean $\pm S D)$ & $14.258 \pm 1.862$ & $14.850 \pm 2.019$ & -1.627 & 0.107 \\
\hline Self-reported craving (mean \pm SD) & $53.970 \pm 16.993$ & $33.015 \pm 16.163$ & 7.368 & 0.000 \\
\hline FTND score $($ mean $\pm \mathrm{SD})$ & $0.485 \pm 0.762$ & $0.441 \pm 0.780$ & 0.333 & 0.739 \\
\hline ADS score $($ mean $\pm \mathrm{SD})$ & $0.103 \pm 0.351$ & $0.177 \pm 0.545$ & -0.935 & 0.351 \\
\hline
\end{tabular}

IGD: Internet gaming disorder; IAT: Internet addiction test, range from 20 to 100; DSM-5: Diagnostic and Statistical Manual of Mental Disorders-5, range from 0 to 9; Gaming playing hours index = [gaming days per week] * [gaming hours per day]; Self-reported cravings, range from 10 to 100; FTND: Fagerström Test for Nicotine Dependence, range from 0 to 10; ADS: Alcohol Dependence Scale, range from 0 to 47 . 


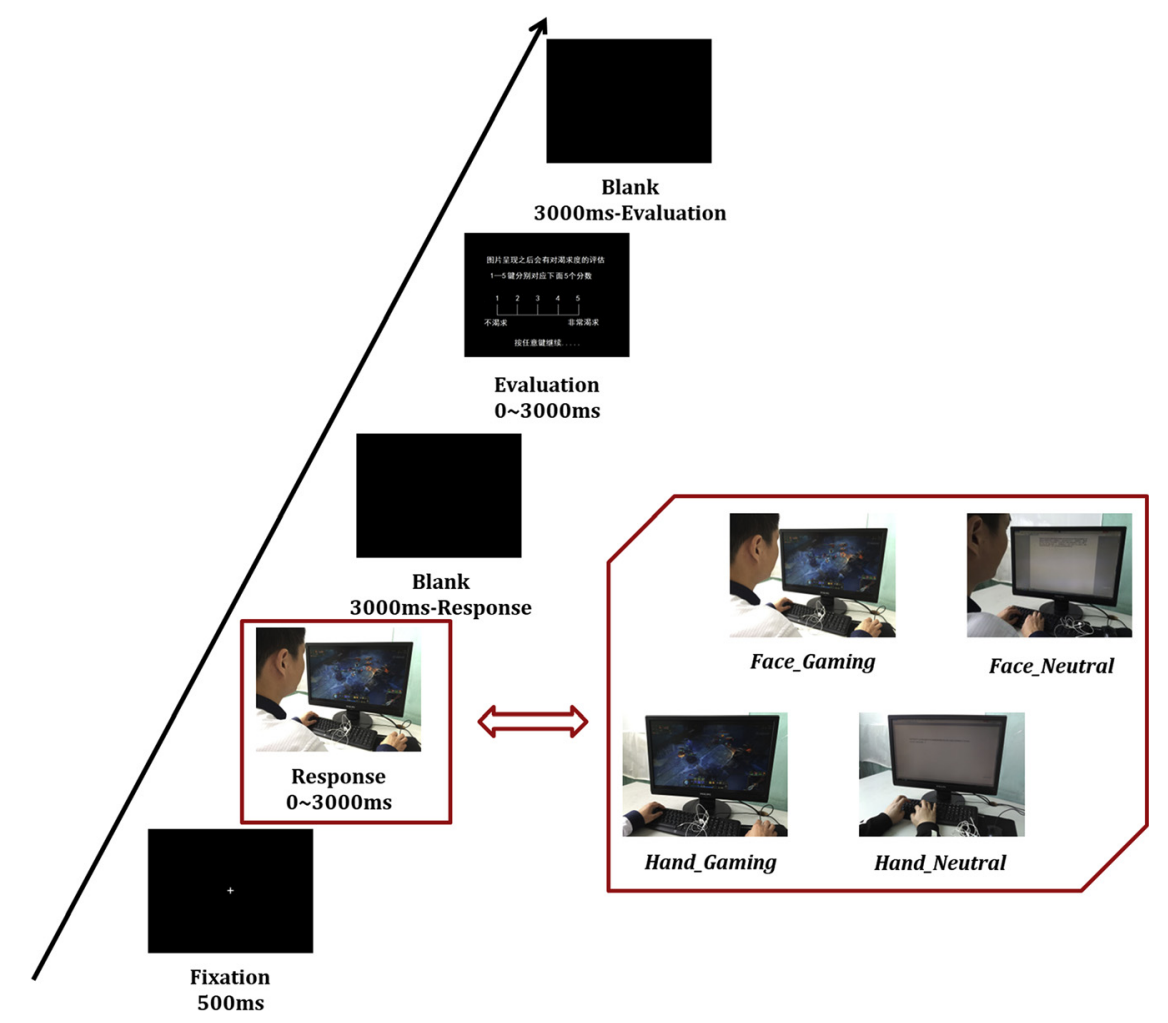

Fig. 1. The cue craving task in the current study. Pictures of an individual playing an online game (showing the player's hands or profile), as well as pictures of typing (showing the individual's hands or profile), served as stimulus cues. After each cue trial, participants rated their craving for the current stimulus

craving) and pressed a button to enter the next trial. A jitter with a random duration (from 1,500 to $3,500 \mathrm{~ms}$ ) is included between each trial. This experiment contained a total of 60 trials (30 gaming cues) and lasted about 9 minutes. After completing the experimental scan, participants would receive a reward of 200 Yuan (about 28 US dollars).

\section{Image acquisition}

All MRI data were acquired using a 3T Siemens Trio MRI scanner. Functional data were collected by a gradient-echo $\mathrm{T} 2{ }^{*}$-weighted gradient-echo echo planar imaging (Pepino et al.) pulse sequence (33 axial slices parallel to the AC-PC line covering the whole brain, $\mathrm{TR}=2,000 \mathrm{~ms}, \mathrm{TE}=30 \mathrm{~ms}$, $\mathrm{FOV}=220 \times 220 \mathrm{~mm}$, flip angle $=90^{\circ}$, matrix $=64 \times 64$, thickness $=3 \mathrm{~mm}, 33 \%$ distance factor). A high-resolution T1-weighted three-dimensional spoiled gradient-recalled structural data were acquired for co-registration with the fMRI images (192 sagittal slices, $\mathrm{TR}=2,530 \mathrm{~ms}$, TE $=2.34$ $\mathrm{ms}$, inversion time $=1,100 \mathrm{~ms}$, FOV $=256 \times 256 \mathrm{~mm}$, flip angle $=7^{\circ}$, matrix $=256 \times 256$, slice thickness $=1 \mathrm{~mm}$, with a $50 \%$ gap).

\section{Image pre-processing}

Spatial pre-processing was performed using SPM12 (https://www.fil.ion.ucl.ac.uk/spm/software/spm12/). Slice timing was performed to correct differences in each slice acquisition time. The motion parameters of individual EPI data were then estimated to correct artefacts, and any subject with motion exceeding $2.5 \mathrm{~mm}$ in directional movement and $2.5^{\circ}$ in rotational movement was excluded from further analysis (None). Corrected images were coregistered with corresponding T1-weighted images and spatially transformed to MNI coordinates. The transformation parameters were applied to the functional images, which resampled to $2 \mathrm{~mm}$ isotropic resolution and finally spatially smoothed by a $6 \mathrm{~mm}$ FWHM isotropic Gaussian kernel.

\section{Region of Interest selection and correlation analysis}

Pre-processing images were modeled using a general linear model, which would convolve the experimental conditions with the canonical hemodynamic response function. For each subject, two conditions (within-subject factors) were defined in the first-level analysis: Gaming cues and Neutral cues, in which trials with incorrect responses were excluded from the design matrix. The matrix parameters were then estimated and contrast condition (Gaming cue vs. Neutral cue) was generated for secondlevel analysis.

To calculate the correlations and the subsequent effective connectivity among the three systems implicated in IGD, we seeded four ROIs: 1) the bilateral putamen; 2) the orbitofrontal cortex (OFC); 3) the bilateral MFG and 4) the bilateral insula (see Fig. 2). These regions met the following criteria: 1) they showed significant activation with a false 
discovery rate (FDR)-corrected cluster threshold $P<0.05$ in the second-level analysis. The group activity map could be found in the supplementary information. Fig. S1 reported regions with significant activation. ; 2) they showed activation in previous fMRI studies using a cue craving task (Courtney, Schacht, Hutchison, Roche, \& Ray, 2016; Zheng et al., 2019; Zilverstand, Huang, Alia-Klein, \& Goldstein, 2018), and 3) they had a theoretical reason to be activated as per both the dual process theory of addiction and the cognitive-behavioral model of IGD (Bechara, 2005; Dong \& Potenza, 2014; He et al., 2019; Kelley \& Berridge, 2002). Details of the anatomical structure contain in each system could be found in the supplementary information.

Volumes of interest (VOIs) were extracted from significantly activated clusters, which were determined by comparison with group-level random effects. The regions where spherical (cluster maxima locations as centers, $8 \mathrm{~mm}$ radius) and cluster mask overlapping was used as the VOI extraction mask. The principal eigenvariate of each VOI extraction was adjusted by the F-contrast of the effects of interest. Each subject generated the corresponding four VOIs (i.e., putamen, OFC, MFG, and insula) for subsequent DCM analysis.
Correlation analysis was used to examine the ROI association changes within the group under different experimental conditions. For each subject, the BOLD signals of the ROIs were separately extracted through the VOI masks. Bonferroni corrections were applied to the correlation results to adjust for family-wise errors $(P<0.002)$. Additionally, the correlations between the BOLD signals of ROIs and behavioral scale, including IAT and craving scores, were also calculated.

\section{Dynamic causal modeling (DCM)}

DCM analysis was performed by DCM12.5 (revision 7487). A detailed description of DCM can be found in K. J. Friston, Harrison, and Penny (2003). Briefly, it is considered a predominant analytical framework for inferring effective connectivity that characterizes the causal influences among neural populations, namely, the mediation effect of one population on another. Detail description of DCM specification could be found in the supplementary information. For the current study, a single "full" model was first specified for each subject after extracting the VOIs. For selecting the

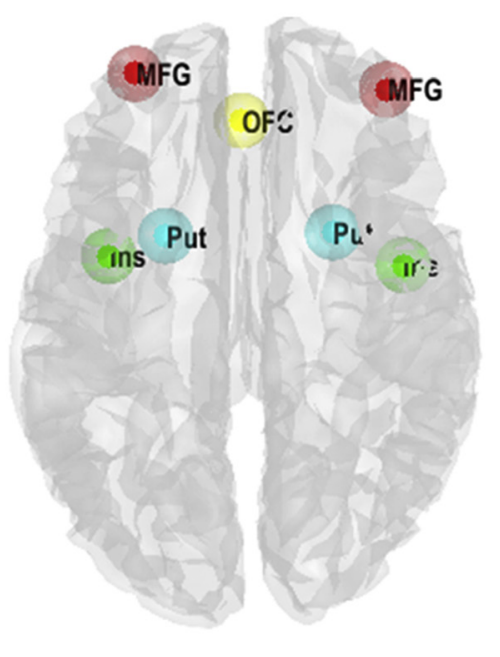

\begin{tabular}{cccc}
\hline Name & Voxels & Location(MNI) & Radius \\
\hline L Insula & 38 & $-39,0,6$ & 8 \\
\hline R Insula & 66 & $45,-3,6$ & 8 \\
\hline L MFG & 26 & $-33,54,99$ & 8 \\
\hline R MFG & 29 & $42,48,9$ & 8 \\
\hline OFC & 24 & $-3,39,-12$ & 8 \\
\hline L Putamen & 60 & $-24,6,3$ & 8 \\
\hline R Putamen & 32 & $24,6,0$ & 8 \\
\hline
\end{tabular}
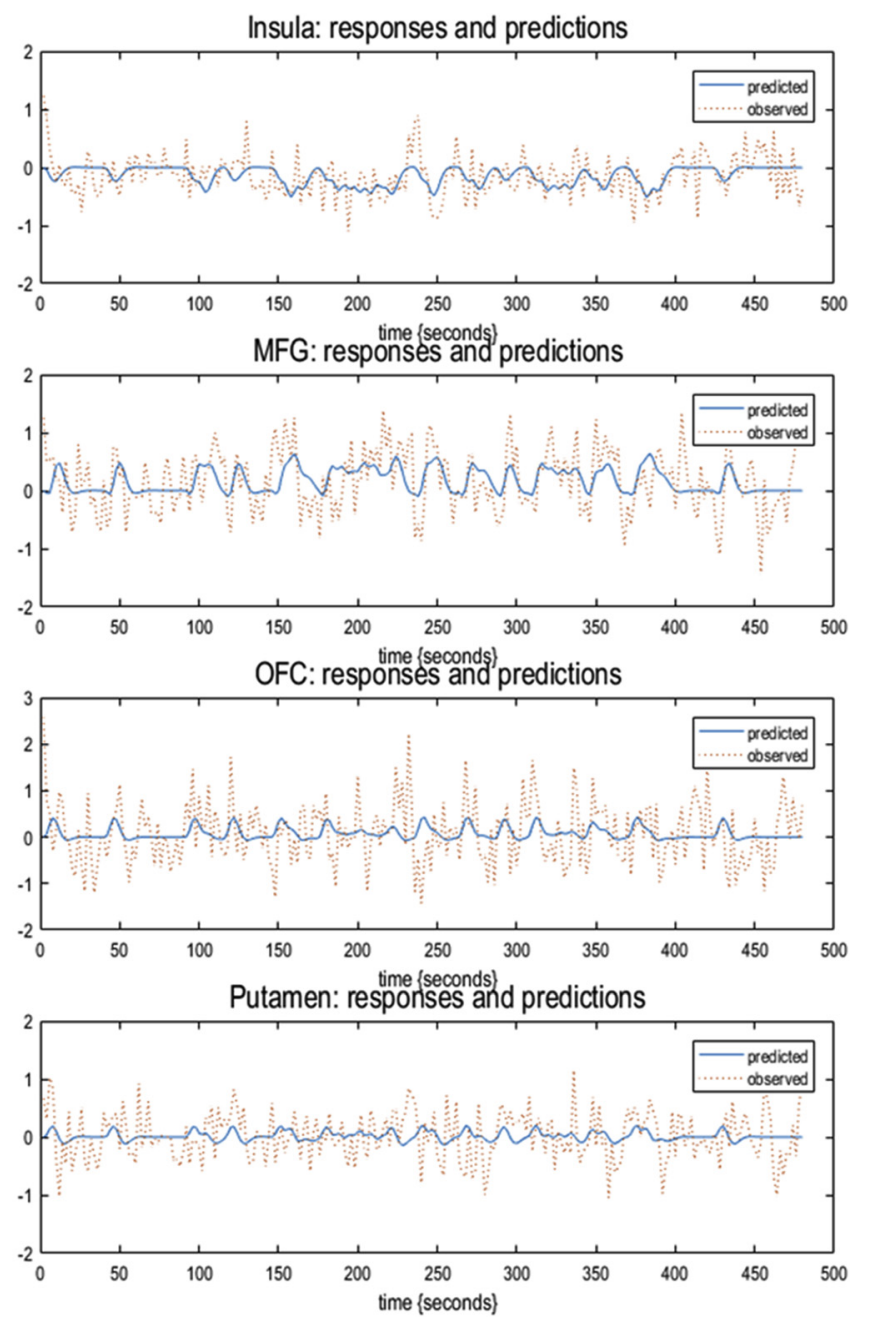

Fig. 2. Illustration of ROIs for DCM. The ROIs included the orbitofrontal cortex (OFC), the bilateral middle frontal gyrus (MFG), the putamen, and the insula. Corresponding time-series are the principal eigenvariates of regions 
driving input and modulation effect, only the gaming cues were designated as experimental inputs for a connected model because the cravings induced by exposure to gaming cues were psychological factors that we were truly interested in. Parameter estimation was conducted by inverting the "full" models for each subject.

After inferring the strength of the model connectivity for each subject, the next step was to quantify the differences between the two groups. To enable differences between groups to be compared efficiently, here the Parametric Empirical Bayes (PEBs) framework was performed to structure hierarchical model over parameters (Friston et al., 2016; Henson, Flandin, Friston, \& Mattout, 2010). It uses the General Linear Model (GLM) to collate the first-level DCM parameters and models at the second level, capturing any unexplained between-subject variability by the covariance component model. In the PEB framework, the group-level design matrix incorporates two regressors, which were coded as 1) the commonalities across subjects and 2) group differences. Since we did not declare any specific a priori models, exploratory Bayesian model reduction was performed to automatically search over the reduced models, after finishing the matrix parameter estimation (Stephan, Penny, Daunizeau, Moran, \& Friston, 2009). A Bayesian model average was then calculated over the 256 models that were obtained by a greedy search. Finally, the resulting parameters were thresholded to focus on the most likely effects, in which threshold based on free energy (with vs. without) was selected and set with posterior probability $>0.99$ (very strong evidence). Also, the correlations between the effective connectivity and behavioral scale, including IAT and craving scores, were also examined. The main analytical pipeline can be seen in Fig. 3.

\section{ETHICS}

This study was approved by the Institutional Review Board of Hangzhou Normal University. All participants signed an informed consent form in accordance with the Declaration of Helsinki.

\section{RESULTS}

\section{Behavioral performance}

Significant differences were observed between the two groups in evoked craving scores (for each subject, craving score corresponding to gaming cues minus score corresponding to neutral cues), of which individuals with IGD showed higher craving to gaming cues than RGUs $(t=2.854, P=0.005$, IGD: $1.861 \pm 1.206$, RGU: $1.270 \pm$ 1.021). No significant group differences were observed in response time $(t=0.862, P=0.390$, IGD: $0.033 \pm 0.174$, RGU: $0.004 \pm 0.216$ ), as well as the accuracy of the two groups' response to cues $(t=-0.778, P=0.438$, IGD: 0.989 \pm 0.177, RGU: $0.991 \pm 0.153)$.

\section{IMAGING RESULTS}

\section{Correlation analysis}

All participants exhibited significant correlations in the following neural pathways when facing gaming cues (see Table 2): 1) the association between the insula and the MFG $(r=0.313, P=0.000) ; 2)$ the association between the

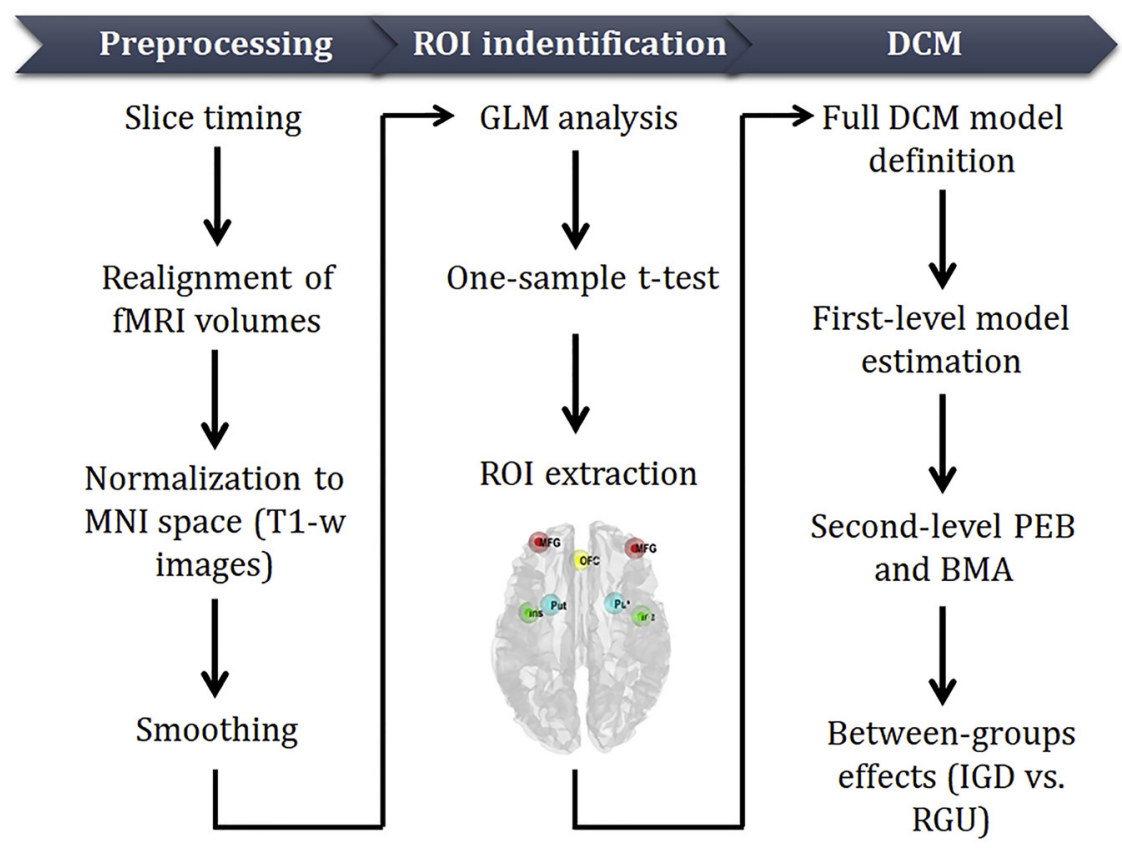

Fig. 3. Analytical pipeline. Analytical pipeline of fMRI data processing for examining effective connectivity within the tripartite systems. Data processing can be subdivided into 3 main steps, highlighted at the top 
Table 2. Correlation results among ROIs

\begin{tabular}{lcccccc}
\hline & \multicolumn{3}{c}{ Gaming cues } & & & \multicolumn{2}{c}{ Neutral cues } \\
\cline { 2 - 7 } Location & RGU & IGD & All & RGU & IGD & All \\
\hline Insula - MFG & $r=0.433^{*}$, & $r=0.202$, & $r=0.313^{*}$, & $r=0.291$, & $r=0.086$, & $r=0.191$, \\
& $P=0.000$ & $P=0.098$ & $P=0.000$ & $P=0.016$ & $P=0.486$ & $P=0.026$ \\
MFG - OFC & $r=0.234$, & $r=0.019$, & $r=0.130$, & $r=0.267$, & $r=-0.019$, & $r=0.135$, \\
& $P=0.055$ & $P=0.877$ & $P=0.131$ & $P=0.028$ & $P=0.878$ & $p=0.117$ \\
OFC - Putamen & $r=0.285$, & $r=0.304$ & $r=0.294^{*}$, & $r=0.128$, & $r=0.078$, & $r=0.106$, \\
& $P=0.018$ & $P=0.012$ & $P=0.001$ & $P=0.298$ & $P=0.527$ & $P=0.219$ \\
Putamen - Insula & $r=0.301$, & $r=-0.055$, & $r=0.110$, & $r=0.219$, & $r=-0.077$, & $r=0.056$, \\
& $P=0.012$ & $P=0.658$ & $P=0.200$ & $P=0.073$ & $P=0.532$ & $P=0.521$ \\
Insula - OFC & $r=-0.121$, & $r=-0.195$, & $r=-0.155$, & $r=0.028$, & $r=-0.145$, & $r=-0.050$, \\
Putamen - MFG & $P=0.327$ & $P=0.110$ & $P=0.072$ & $P=0.819$ & $P=0.238$ & $P=0.564$ \\
& $r=0.400^{*}$, & $r=0.141$, & $r=0.258^{*}$, & $r=0.104$, & $r=0.185$, & $r=0.142$, \\
& $P=0.001$ & $P=0.250$ & $P=0.002$ & $P=0.397$ & $P=0.131$ & $P=0.099$ \\
\hline
\end{tabular}

*: significant connectivity after Bonferroni multiple comparison correction,

$P<0.002$.
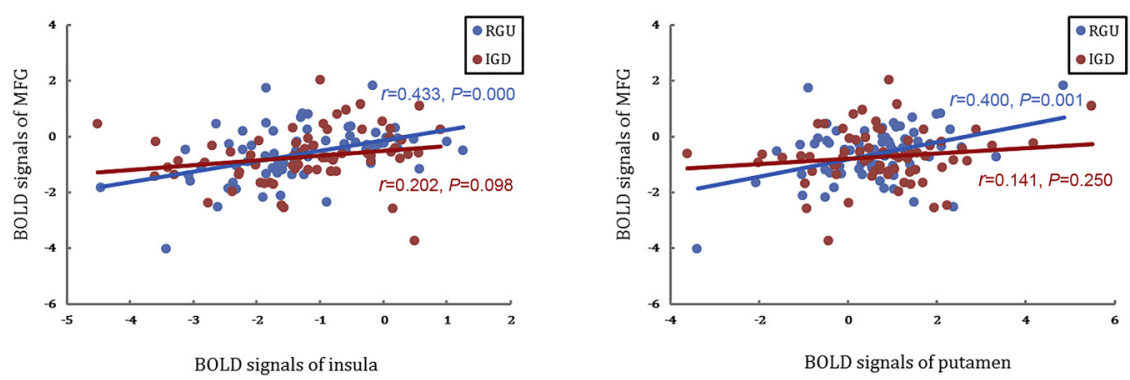

Fig. 4. Correlation results during the cue craving task. The figure shows the correlation between MFG and insula (left image)/MFG (right image) activity when the two groups were exposed to gaming cues

Table 3. Group differences by Parametric Empirical Bayes inference

\begin{tabular}{|c|c|c|c|c|c|}
\hline Location & Strength $(\mathrm{Hz})$ & Posterior probability & Location & Strength $(\mathrm{Hz})$ & $\begin{array}{l}\text { Posterior } \\
\text { probability }\end{array}$ \\
\hline \multicolumn{6}{|c|}{ Endogenous connection } \\
\hline Insula $\rightarrow$ Insula & -0.001 & 0 & $\mathrm{OFC} \rightarrow$ Insula & -0.007 & 0 \\
\hline Insula $\rightarrow$ MFG & 0.066 & 0.99 & $\mathrm{OFC} \rightarrow \mathrm{MFG}$ & -0.002 & 0 \\
\hline Insula $\rightarrow$ OFC & 0.002 & 0 & $\mathrm{OFC} \rightarrow \mathrm{OFC}$ & 0.003 & 0 \\
\hline Insula $\rightarrow$ Putamen & 0.062 & 0.99 & OFC $\rightarrow$ Putamen & -0.008 & 0 \\
\hline MFG $\rightarrow$ Insula & 0.006 & 0 & Putamen $\rightarrow$ Insula & 0.002 & 0 \\
\hline $\mathrm{MFG} \rightarrow \mathrm{MFG}$ & -0.137 & 0.99 & Putamen $\rightarrow$ MFG & -0.070 & 0.99 \\
\hline $\mathrm{MFG} \rightarrow \mathrm{OFC}$ & 0 & 0 & Putamen $\rightarrow$ OFC & 0.083 & 0.99 \\
\hline MFG $\rightarrow$ Putamen & -0.005 & 0 & Putamen $\rightarrow$ Putamen & 0.051 & 0.73 \\
\hline \multicolumn{6}{|c|}{ Modulation effect of gaming cues } \\
\hline Insula $\rightarrow$ Insula & -0.026 & 0 & $\mathrm{OFC} \rightarrow$ Insula & 0.039 & 0 \\
\hline Insula $\rightarrow$ MFG & 0.042 & 0 & $\mathrm{OFC} \rightarrow \mathrm{MFG}$ & 0.054 & 0 \\
\hline Insula $\rightarrow$ OFC & 0.220 & 0.62 & $\mathrm{OFC} \rightarrow \mathrm{OFC}$ & 0.013 & 0 \\
\hline Insula $\rightarrow$ Putamen & -0.023 & 0 & OFC $\rightarrow$ Putamen & 0.060 & 0 \\
\hline $\mathrm{MFG} \rightarrow$ Insula & -0.052 & 0 & Putamen $\rightarrow$ Insula & -0.057 & 0 \\
\hline $\mathrm{MFG} \rightarrow \mathrm{MFG}$ & 0.535 & 0.99 & Putamen $\rightarrow$ MFG & 0.267 & 0.74 \\
\hline $\mathrm{MFG} \rightarrow \mathrm{OFC}$ & 0.046 & 0 & Putamen $\rightarrow$ OFC & 0.005 & 0 \\
\hline MFG $\rightarrow$ Putamen & -0.022 & 0 & Putamen $\rightarrow$ Putamen & 0.010 & 0 \\
\hline \multicolumn{6}{|c|}{ Driving input effect of gaming cues } \\
\hline Insula & -0.013 & 0 & OFC & 0.011 & 0 \\
\hline MFG & -0.007 & 0 & Putamen & 0.016 & 0 \\
\hline
\end{tabular}

Posterior mean strength $(\mathrm{Hz})$ and posterior probability of all parameters implicated in group differences, including endogenous connection, modulation and driving input effect, are outlined. 
putamen and the MFG $(r=0.258, P=0.002)$ and 3$)$ the association between the putamen and the OFC $(r=0.294$, $P=0.001)$. After grouping, there was no significant correlation observed among the 4 ROIs in either the individuals with IGD or the RGUs when facing neutral cues. The RGUs still exhibited significant positive correlations under gaming cues in the following neural pathways: 1) the insula-MFG $(r=0.433, P=0.000)$ and 2$)$ the putamen-MFG $(r=0.400$, $P=0.001$, see Fig. 4). The same correlations were not significant in the individuals with IGD: 1$)$ the insula-MFG $(r=0.202, P=0.098)$ and 2$)$ the putamen-MFG $(r=0.141$, $P=0.250)$. In addition, the IAT scores of IGD individuals were positively associated with their BOLD signal in the putamen when facing gaming cues $(r=0.299, P=0.013$, uncorrected).

\section{DCM results}

Second level analysis with PEB revealed effective connectivity difference between the individuals with IGD and the RGUs. The parameters that were used to characterize the difference between groups are given in Table 3, which shows the posterior probability and strength of all parameters. Fig. 5 illustrates parameters that have a posterior probability greater than 0.99. Specifically, the negative effect in the connection from the putamen to the MFG indicates that putamen exhibits more inhibitory regulation on the MFG in IGD than in regular gaming use, whereas the positive effects in 1) the connection from the insula to the MFG, 2) the connection from the putamen to the OFC and 3) the connection from the insula to the putamen indicate the increased regulation of excitability in these pathways in IGD. Moreover, the negative self-connectivity of the MFG indicates increased self-inhibition in IGD, which was more robustly modulated by gaming cues. Additionally, the correlation results showed that the craving scores of IGD individuals were negatively associated with their effective connectivity from the putamen to the MFG $(r=-0.310$, $P=0.010$, uncorrected).

\section{DISCUSSION}

The present study investigated the dynamic relationship underlying three systems in IGD and provided new insights into the potential imbalance of the tripartite system in addicted individuals. Specifically, using correlations and DCM analyses, the results found that RGUs showed a significant positive correlation in the neural pathways connecting the putamen-MFG-insula when facing gaming cues, which is missing in individuals with IGD. Additionally, the difference in effective connectivity patterns between groups showed an inhibitory effective connection from the putamen to the MFG in IGD, which may prompt hypoactivation in the control system, and this effective connectivity was negatively associated with craving scores in IGD. Meanwhile, IGD also demonstrated increased excitatory neuromodulation in the effective connections among the insulaputamen-OFC, a neural pathway involving reward-related activity.

\section{High coupling in the putamen-MFG-insula pathway ensures a balance among the tripartite systems}

The results showed that the reward, executive control, and interoceptive awareness systems are highly coupled in RGUs when exposed to reward stimuli, while the association does not work in individuals with IGD, which reveals that

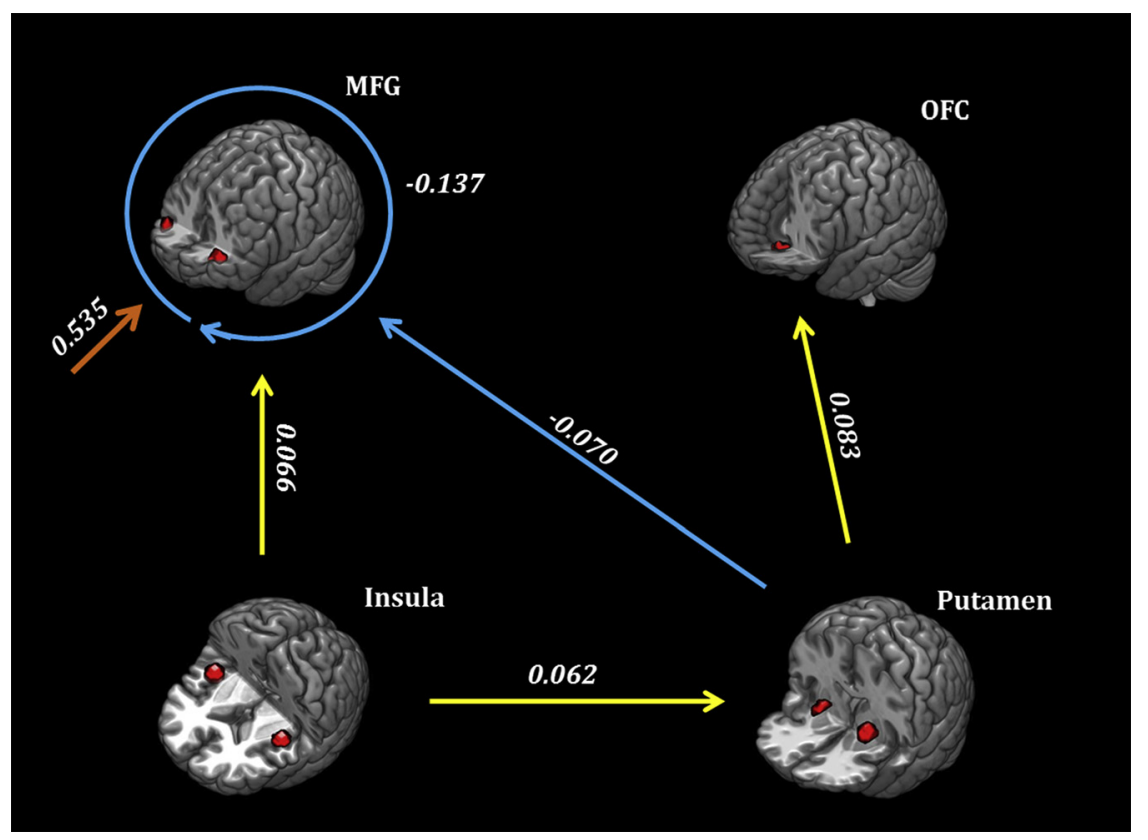

Fig. 5. Group-level Inference with Parametric Empirical Bayes framework. The image shows a deviation in effective connectivity between the two groups. Arrows indicate directions of the connections, and values indicate connection strength 
positive correlations among the tripartite activities may be the major foundation for maintaining relative balances among the systems. This is complementary with previous research results showing that functional connectivity between the striatum and the prefrontal cortex (Becker et al., 2017; Forbes, Rodriguez, Musselman, \& Narendran, 2014), as well as between the insula and prefrontal cortex (Dong, Liu, et al., 2019a; Li et al., 2019), are reduced in addicted individuals, reflecting enhanced internal craving and reward sensitivity as well as weak executive control. In addition, given that these couplings among systems are only observed in healthy groups, we infer that it may be an important marker for predicting the risk of addiction and even play a causal role in the transition to IGD. Indeed, several studies have suggested that neural pathways involving these systems could predict the development of addiction-related problems independently (Heitzeg, Cope, Martz, \& Hardee, 2015; Norman et al., 2011; Wetherill, Squeglia, Yang, \& Tapert, 2013; Whelan et al., 2014). The present results extend such findings and show that the interactions among these systems are also worth attention in addiction studies, as individuals who show imbalances among these neural systems are more likely at potential risk of addiction.

\section{Inhibitory neuromodulation of the putamen to MFG disrupts the balance among systems in IGD}

It is worth noting that the current study not only calculated the correlation among systems, but also performed DCM analysis to support the causal inference between group-level models. DCM results show an inhibitory effective connection from the putamen to the MFG in IGD, which reflects that the reward system of individuals with IGD exerts a robust bottom-up driving effect over their control system. Specifically, the basal ganglion network (i.e., reward system) is indispensable for the incentive motivational effects for both consuming rewards (e.g., drugs, foods) and engaging in rewarding activities (e.g., online game, sex) (Boileau et al., 2003; Kringelbach, 2005). The normal population possesses a flexible cognitive control system for making the decision and resisting rewards that can lead to negative consequences in the long term, indicative of a top-down regulation of the reward system (Noel et al., 2013). However, this feature seems to be reverse in addicted individuals, who show a loss of cognitive control over addiction cues, as well as excessive reward-seeking behavior. For this potential risk, our view is consistent with previous studies that report that these problem behaviors may be caused by the mechanisms underlying the negative correlation between reward processing and prefrontal inhibition (Weafer et al., 2019), meanwhile, the present results further reveal that the reward system exhibits a powerful drive to inhibit the control system during the process of reward. Although it is rarely mentioned, these results reflect the bottom-up mechanism in the dual-process theory of addiction (Bechara, 2005), which may reveal a totally specific feature for IGD as a behavioral addiction, namely the gaming cues can trigger bottom-up involuntary signal from the reward system that hijacks the cognitive resources necessary for normal operation of the control system and weakens the capacity to resist the gaming craving. Here, we highlight the driving role of reward systems in the tripartite system. Indeed, both abnormal cognitive control and reward sensitivity in addicted individuals may also be regulated by their reduced striatal dopamine D2 receptors; these pieces of evidence all point to reveal the significance of the reward system in addiction research (Ghahremani et al., 2012; Lee et al., 2009; Pepino et al., 2016). Future multimodal imaging studies should attempt to combine PET and fMRI to explore the underlying association between D2 receptors and inhibitory neuromodulation of reward systems.

Additionally, it should be noted that the reward system in the present study is mainly located in the part of the bilateral putamen subordinate to the dorsal striatum. Although the different contributions of the dorsal and ventral striatum in response to reward activity have been identified by other studies, increasing evidence suggests that there is a tighter association between the dorsal striatum and the prefrontal cortex (Pelchat, Johnson, Chan, Valdez, \& Ragland, 2004; Rothemund et al., 2007; Zhou et al., 2018). Nevertheless, current results indicate that an inhibitory effective connection from the putamen to the MFG in IGD may be a risk factor contributing to system imbalance.

\section{Excitatory neuromodulation of the insula-putamen-OFC pathway enhances the reward-related regional associations in IGD}

The DCM results also show increased excitatory neuromodulation in the effective connections of the insula-putamen-OFC pathway in individuals with IGD relative to RGUs. Whereas the OFC is located below the prefrontal cortex, this region often shows stronger connectivity with the reward system (Huckins et al., 2019; Ma et al., 2010). Indeed, animal model studies have shown that reward stimuli are associated with activation of both mesolimbic dopamine brain regions (i.e., the ventral striatum) and the OFC (Boileau et al., 2003), and human studies further suggest that this reward-related activity also extends into the dorsal striatum, medial prefrontal cortex, and insula (Gottfried, O’Doherty, \& Dolan, 2003; Kringelbach et al., 2003). Therefore, the increased excitatory neuromodulation observed in the insula-putamen-OFC pathway may be associated with a strong response of IGD to cue signals, in which the insula converts the subjective experience of craving into an impulse signal and then further enhances neuromodulation to the downstream regions associated with reward-seeking.

Finally, an excitatory effect from the insula to the MFG was also observed in current study. According to the threesystems neural model of addiction proposed by Noel et al. (2013), the insula is hypothesized as a neural system that increase reward drivers and weaken cognitive control in addicts. The current results seem to be inconsistent with this assumption to some extent. One possible explanation is that these participants need to make correct key press responses 
based on pictures during the task, so they have to make certain cognitive efforts to successfully complete the task. However, inhibitory effects from the reward system may impair the cognitive functional integrity of the control system, affecting task performance. Here the excitatory effect from the insula to the MFG may serve as a compensatory regulation to maintain task performance of IGD group. However, it should be noted that the current evidence is too preliminary to support a complete inference. Thus, further work is needed to determine the specificity of these findings to inter-system regulation.

Understanding the underlying imbalance among systems in IGD is conducive to informing the development of new therapeutic strategies that can apply to, not only IGD but perhaps other addiction and impulsive disorders. The current study demonstrated that high coupling among the tripartite systems may be the major foundation for maintaining relative balances among systems and the inhibitory neuromodulation of the putamen to the MFG in IGD may be a potential risk factor contributing to the imbalance. These results extend several prospective studies ( $\mathrm{He}$ et al., 2019; Weafer et al., 2019) exploring the neural association between systems and have crucial implications for understanding potential risks for addictive disorders, particularly in individuals with overactive reward functioning. We suggest that future clinical studies should further assess the neuromodulation by the reward system to the control system, which will provide a critical biomarker for prevention and treatment efforts in the high-risk population.

\section{LIMITATIONS}

Some limitations should be mentioned. First, whereas the main conclusion relies on DCM results, the mechanisms underlying both the self-inhibition in the MFG and its experimental modulation remain unclear. Indeed, individual self-connections often show inhibition, including studies of both task-based DCM and resting state DCM (i.e., spectral DCM) (Hagmann et al., 2008; Razi, Kahan, Rees, \& Friston, 2015; Wang, Zheng, Du, \& Dong, 2019), but there is no clear discussion about this inhibition currently. We suggest that future research should further evaluate the implications of inhibitory self-connections, which may contain a wealth of important information. Second, we observed a marginal significance on sex difference between two groups in the current study. Although we included sex as a covariate in the subsequent brain imaging analysis to control the impact of sex effects on the data results, future research should note this issue. Third, the mean and duration of inter-stimulusinterval (ISI) in event-related designs affect statistical efficiency. Although Dale (1999) suggest that for shorter mean ISIs (e.g less than 6s), variable ISI designs would increase dramatically the efficiency, we did not optimized the parameters of ISI through efficiency estimation in the current study, which may prevent current experimental designs from achieving optimal statistical efficiency. Fourth, current results showed an activation pattern that is inconsistent with previous studies (Ko et al., 2009; Vollstadt-Klein et al., 2010, 2011). One possible explanation is that the specificity of different types of addictions causes these deviations. In addition, subjects' inclusion criteria may also be a potential factor. Anyway, more comparative research is needed in the future to determine these differences. Finally, we did not examine the neurodynamics among systems under spontaneous activity, namely, resting state scans. We suggest that future research needs to determine whether these systems are coupled without any task manipulation.

\section{CONCLUSIONS}

The current study showed that coupling in the putamenMFG-insula pathway is missing in the IGD when facing addictive cues, which might be a potential characterization of relative imbalance among the tripartite systems in IGD. Further DCM results showed an inhibitory effective connection from the putamen to the MFG in IGD, suggesting that an impaired executive control function might be caused by this inhibitory neuromodulation. Given that prospective studies have demonstrated negative correlations between the prefrontal cortex-striatum circuit, the current study extended these studies and highlighted the inhibitory neuromodulation of the reward system to the control system, which might partly explain how addictive disorder impairs executive control and contributes to imbalances among the tripartite systems.

Funding sources: This research was supported by Zhejiang Provincial Science Foundation (LY20C090005).

Authors' contribution: Min Wang analyzed the data and wrote the first draft of the manuscript. Haohao Dong was responsible for analyzing the partial data. Xiaoxia Du and Hui Zheng contributed to fMRI data collection. GuangHeng Dong designed this research and edited the manuscript. All authors contributed to and approved the final manuscript.

Conflict of interest: The authors declare that no competing interests exist.

\section{APPENDIX A. SUPPLEMENTARY DATA}

Supplementary data to this article can be found online at https://doi.org/10.1556/2006.2020.00029.

\section{REFERENCES}

Bari, A., \& Robbins, T. W. (2013). Inhibition and impulsivity: Behavioral and neural basis of response control. Progress in 
Neurobiology, 108, 44-79. https://doi.org/10.1016/j.pneurobio. 2013.06.005.

Bechara, A. (2005). Decision making, impulse control and loss of willpower to resist drugs: A neurocognitive perspective. Nature Neuroscience, 8(11), 1458-1463. https://doi.org/10. 1038/nn1584.

Becker, A., Kirsch, M., Gerchen, M. F., Kiefer, F., \& Kirsch, P. (2017). Striatal activation and frontostriatal connectivity during non-drug reward anticipation in alcohol dependence. Addiction Biology, 22(3), 833-843. https://doi.org/10.1111/adb.12352.

Boileau, I., Assaad, J. M., Pihl, R. O., Benkelfat, C., Leyton, M., Diksic, M., et al. (2003). Alcohol promotes dopamine release in the human nucleus accumbens. Synapse (New York, N.Y.), 49(4), 226-231. https://doi.org/10.1002/syn.10226.

Chang, M. K., \& Law, S. P. M. (2008). Factor structure for young's internet addiction test: A confirmatory study. Computers in Human Behavior, 24(6), 2597-2619. https://doi.org/10.1016/j. chb.2008.03.001.

Courtney, K. E., Schacht, J. P., Hutchison, K., Roche, D. J. O., \& Ray, L. A. (2016). Neural substrates of cue reactivity: Association with treatment outcomes and relapse. Addiction Biology, 21(1), 3-22. https://doi.org/10.1111/adb.12314.

Dale, A. M. (1999). Optimal experimental design for eventrelated fMRI. Human Brain Mapping, 8(2-3), 109-114. https://doi. org/10.1002/(sici)1097-0193(1999)8:2/3<109::aid-hbm7>3.0.co;2-w.

Dong, G., Liu, X., Zheng, H., Du, X., \& Potenza, M. N. (2019a). Brain response features during forced break could predict subsequent recovery in internet gaming disorder: A longitudinal study. Journal of Psychiatric Research, 113, 17-26. https:// doi.org/10.1016/j.jpsychires.2019.03.003.

Dong, G., \& Potenza, M. N. (2014). A cognitive-behavioral model of Internet gaming disorder: Theoretical underpinnings and clinical implications. Journal of Psychiatric Research, 58, 7-11. https://doi.org/10.1016/j.jpsychires.2014.07.005.

Dong, G., Wang, L., Du, X., \& Potenza, M. N. (2017). Gaming increases craving to gaming-related stimuli in individuals with internet gaming disorder. Biological Psychiatry. Cognitive neuroscience and neuroimaging, 2(5), 404-412. https://doi.org/ 10.1016/j.bpsc.2017.01.002.

Dong, G., Wang, M., Liu, X., Liang, Q., Du, X., \& Potenza, M. N. (2019b). Cue-elicited craving-related lentiform activation during gaming deprivation is associated with the emergence of Internet gaming disorder. Addiction Biology, 25(1), e12713. https://doi.org/10.1111/adb.12713.

Feil, J., Sheppard, D., Fitzgerald, P. B., Yuecel, M., Lubman, D. I., \& Bradshaw, J. L. (2010). Addiction, compulsive drug seeking, and the role of frontostriatal mechanisms in regulating inhibitory control. Neuroscience \& Biobehavioral Reviews, 35(2), 248-275. https://doi.org/10.1016/j.neubiorev.2010.03.001.

Forbes, E. E., Rodriguez, E. E., Musselman, S., \& Narendran, R. (2014). Prefrontal response and frontostriatal functional connectivity to monetary reward in abstinent alcohol-dependent young adults. PLoS One, 9(5). https://doi.org/10.1371/journal. pone.0094640.

Friston, K. J., Harrison, L., \& Penny, W. (2003). Dynamic causal modelling. Neuroimage, 19(4), 1273-1302. https://doi.org/10. 1016/s1053-8119(03)00202-7.
Friston, K. J., Litvak, V., Oswal, A., Razi, A., Stephan, K. E., van Wijk, B. C. M., et al. (2016). Bayesian model reduction and empirical Bayes for group (DCM) studies. NeuroImage, 128, 413-431. https://doi.org/10.1016/j.neuroimage.2015.11.015.

Ghahremani, D. G., Lee, B., Robertson, C. L., Tabibnia, G., Morgan, A. T., De Shetler, N., et al. (2012). Striatal dopamine D-2/D-3 receptors mediate response inhibition and related activity in frontostriatal neural circuitry in humans. Journal of Neuroscience, 32(21), 7316-7324. https://doi.org/10.1523/jneurosci. 4284-11.2012.

Gottfried, J. A., O'Doherty, J., \& Dolan, R. J. (2003). Encoding predictive reward value in human amygdala and orbitofrontal cortex. Science (New York, N.Y.), 301(5636), 1104-1107. https:// doi.org/10.1126/science.1087919.

Grant, J. E., Potenza, M. N., Weinstein, A., \& Gorelick, D. A. (2010). Introduction to behavioral addictions. The American Journal of Drug and Alcohol Abuse, 36(5), 233-241. https://doi. org/10.3109/00952990.2010.491884.

Hagmann, P., Cammoun, L., Gigandet, X., Meuli, R., Honey, C. J., Wedeen, V. J., et al. (2008). Mapping the structural core of human cerebral cortex. PLoS Biology, 6(7), 1479-1493. https:// doi.org/10.1371/journal.pbio.0060159.

He, Q., Huang, X., Zhang, S., Turel, O., Ma, L., \& Bechara, A. (2019). Dynamic causal modeling of insular, striatal, and prefrontal cortex activities during a food-specific go/NoGo task. Biological Psychiatry. Cognitive Neuroscience and Neuroimaging, 4(12), 1080-1089. https://doi.org/10.1016/j.bpsc.2018. 12.005 .

Heitzeg, M. M., Cope, L. M., Martz, M. E., \& Hardee, J. E. (2015). Neuroimaging risk markers for substance abuse: Recent findings on inhibitory control and reward system functioning. Current Addiction Reports, 2(2), 91-103.

Henson, R. N., Flandin, G., Friston, K. J., \& Mattout, J. (2010). A parametric empirical bayesian framework for fMRI-constrained MEG/EEG source reconstruction. Human Brain Mapping, 31(10), 1512-1531. https://doi.org/10.1002/hbm. 20956.

Huckins, J. F., Adeyemo, B., Miezin, F. M., Power, J. D., Gordon, E. M., Laumann, T. O., et al. (2019). Reward-related regions form a preferentially coupled system at rest. Human Brain Mapping, 40(2), 361-376. https://doi.org/10.1002/hbm.24377.

Kelley, A. E., \& Berridge, K. C. (2002). The neuroscience of natural rewards: Relevance to addictive drugs. Journal of Neuroscience: The Official Journal of the Society for Neuroscience, 22(9), 3306-3311.

Ko, C. H., Liu, G. C., Hsiao, S., Yen, J. Y., Yang, M. J., Lin, W. C., et al. (2009). Brain activities associated with gaming urge of online gaming addiction. Journal of Psychiatric Research, 43(7), 739-747. https://doi.org/10.1016/j.jpsychires.2008.09.012.

Koob, G. F., \& Le Moal, M. (2001). Drug addiction, dysregulation of reward, and allostasis. Neuropsychopharmacology: Official Publication of the American College of Neuropsychopharmacology, 24(2), 97-129. https://doi.org/10.1016/ s0893-133x (00)00195-0.

Kringelbach, M. L. (2005). The human orbitofrontal cortex: Linking reward to hedonic experience. Nature Reviews. Neuroscience, 6(9), 691-702. https://doi.org/10.1038/nrn1747. 
Kringelbach, M. L., O’Doherty, J., Rolls, E. T., \& Andrews, C. (2003). Activation of the human orbitofrontal cortex to a liquid food stimulus is correlated with its subjective pleasantness. Cerebral cortex (New York, N.Y.: 1991), 13(10), 1064-1071. https://doi.org/10.1093/cercor/13.10.1064.

Kuss, D. J. (2013). Internet gaming addiction: Current perspectives. Psychology Research and Behavior Management, 6, 125-137. https://doi.org/10.2147/prbm.s39476.

Kuss, D. J., \& Griffiths, M. D. (2012). Internet and gaming addiction: A systematic literature review of neuroimaging studies. Brain Sciences, 2(3), 347-374. https://doi.org/10.3390/ brainsci2030347.

Kuss, D. J., Griffiths, M. D., \& Binder, J. F. (2013). Internet addiction in students: Prevalence and risk factors. Computers in Human Behavior, 29(3), 959-966. https://doi.org/10.1016/j.chb. 2012.12.024.

Kuss, D. J., Griffiths, M. D., \& Pontes, H. M. (2017). Chaos and confusion in DSM-5 diagnosis of internet gaming issues, concerns, and recommendations for clarity in the field. Journal of Behavioral Addictions, 6(2), 103-109. https://doi.org/10.1556/ 2006.5.2016.062.

Kuss, D. J., Pontes, H. M., \& Griffiths, M. D. (2018). Neurobiological correlates in internet gaming disorder: A systematic literature review. Frontiers in Psychiatry, 9, 166. https://doi.org/ 10.3389/fpsyt.2018.00166.

Lee, B., London, E. D., Poldrack, R. A., Farahi, J., Nacca, A., Monterosso, J. R., et al. (2009). Striatal dopamine D-2/D-3 receptor availability is reduced in methamphetamine dependence and is linked to impulsivity. Journal of Neuroscience, 29(47), 14734-14740. https://doi.org/10.1523/jneurosci.3765-09.2009.

Li, X., Chen, L., Ma, R., Wang, H., Wan, L., Wang, Y., et al. (2019). The top-down regulation from the prefrontal cortex to insula via hypnotic aversion suggestions reduces smoking craving. Human Brain Mapping, 40(6), 1718-1728. https://doi.org/10. 1002/hbm.24483.

Luijten, M., Machielsen, M. W. J., Veltman, D. J., Hester, R., de Haan, L., \& Franken, I. H. A. (2014). Systematic review of ERP and fMRI studies investigating inhibitory control and error processing in people with substance dependence and behavioural addictions. Journal of Psychiatry \& Neuroscience, 39(3), 149-169. https://doi.org/10.1503/jpn.130052.

Ma, N., Liu, Y., Li, N., Wang, C. X., Zhang, H., Jiang, X. F., et al. (2010). Addiction related alteration in resting-state brain connectivity. Neuroimage, 49(1), 738-744. https://doi.org/10. 1016/j.neuroimage.2009.08.037.

Motzkin, J. C., Baskin-Sommers, A., Newman, J. P., Kiehl, K. A., \& Koenigs, M. (2014). Neural correlates of substance abuse: Reduced functional connectivity between areas underlying reward and cognitive control. Human Brain Mapping, 35(9), 4282-4292. https://doi.org/10.1002/hbm.22474.

Noel, X., Brevers, D., \& Bechara, A. (2013). A neurocognitive approach to understanding the neurobiology of addiction. Current Opinion in Neurobiology, 23(4), 632-638. https://doi. org/10.1016/j.conb.2013.01.018.

Norman, A. L., Pulido, C., Squeglia, L. M., Spadoni, A. D., Paulus, M. P., \& Tapert, S. F. (2011). Neural activation during inhibition predicts initiation of substance use in adolescence. Drug and Alcohol Dependence, 119(3), 216-223. https://doi.org/10. 1016/j.drugalcdep.2011.06.019.

Pelchat, M. L., Johnson, A., Chan, R., Valdez, J., \& Ragland, J. D. (2004). Images of desire: Food-craving activation during fMRI. NeuroImage, 23(4), 1486-1493. https://doi.org/10.1016/j. neuroimage.2004.08.023.

Pepino, M. Y., Eisenstein, S. A., Bischoff, A. N., Klein, S., Moerlein, S. M., Perlmutter, J. S., et al. (2016). Sweet dopamine: Sucrose preferences relate differentially to striatal D-2 receptor binding and age in obesity. Diabetes, 65(9), 2618-2623. https://doi.org/ 10.2337/db16-0407.

Petry, N. M., Rehbein, F., Gentile, D. A., Lemmens, J. S., Rumpf, H.J., Moessle, T., et al. (2014). An international consensus for assessing internet gaming disorder using the new DSM-5 approach. Addiction, 109(9), 1399-1406. https://doi.org/10. 1111/add.12457.

Petry, N. M., Zajac, K., \& Ginley, M. K. (2018). Behavioral addictions as mental disorders: To be or not to be? In T. Widiger \& T. D. Cannon (Eds.), Annual review of clinical psychology, (Vol. 14, pp. 399-423).

Razi, A., Kahan, J., Rees, G., \& Friston, K. J. (2015). Construct validation of a DCM for resting state fMRI. Neuroimage, 106, 1-14. https://doi.org/10.1016/j.neuroimage.2014.11.027.

Rothemund, Y., Preuschhof, C., Bohner, G., Bauknecht, H.-C., Klingebiel, R., Flor, H., et al. (2007). Differential activation of the dorsal striatum by high-calorie visual food stimuli in obese individuals. NeuroImage, 37(2), 410-421. https://doi.org/10. 1016/j.neuroimage.2007.05.008.

Sescousse, G., Caldu, X., Segura, B., \& Dreher, J. C. (2013). Processing of primary and secondary rewards: A quantitative metaanalysis and review of human functional neuroimaging studies. Neuroscience \& Biobehavioral Reviews, 37(4), 681-696. https:// doi.org/10.1016/j.neubiorev.2013.02.002.

Sheehan, D. V., Lecrubier, Y., Sheehan, K. H., Amorim, P., Janavs, J., Weiller, E., et al. (1998). The mini-international neuropsychiatric interview (M.I.N.I.): The development and validation of a structured diagnostic psychiatric interview for DSM-IV and ICD-10. Journal of Clinical Psychiatry, 59 (Suppl. 20), 22-33; quiz 34-57. https://doi.org/10.4088/JCP.09m05305whi.

Somerville, L. H., Hare, T., \& Casey, B. J. (2011). Frontostriatal maturation predicts cognitive control failure to appetitive cues in adolescents. Journal of Cognitive Neuroscience, 23(9), 21232134. https://doi.org/10.1162/jocn.2010.21572.

Spechler, P. A., Chaarani, B., Hudson, K. E., Potter, A., Foxe, J. J., \& Garavan, H. (2016). Response inhibition and addiction medicine: From use to abstinence. Progress in Brain Research, 223, 143-164. https://doi.org/10.1016/bs.pbr.2015.07.024.

Stephan, K. E., Penny, W. D., Daunizeau, J., Moran, R. J., \& Friston, K. J. (2009). Bayesian model selection for group studies. NeuroImage, 46(4), 1004-1017. https://doi.org/10. 1016/j.neuroimage.2009.03.025.

Volkow, N. D., \& Baler, R. D. (2015). NOW vs LATER brain circuits: Implications for obesity and addiction. Trends in Neurosciences, 38(6), 345-352. https://doi.org/10.1016/j.tins.2015. 04.002 .

Volkow, N. D., \& Wise, R. A. (2005). How can drug addiction help us understand obesity? Nature Neuroscience, 8(5), 555-560. https://doi.org/10.1038/nn1452. 
Vollstadt-Klein, S., Loeber, S., Kirsch, M., Bach, P., Richter, A., Buhler, M., et al. (2011). Effects of cue-exposure treatment on neural cue reactivity in alcohol dependence: A randomized trial. Biological Psychiatry, 69(11), 1060-1066. https://doi.org/10. 1016/j.biopsych.2010.12.016.

Vollstadt-Klein, S., Wichert, S., Rabinstein, J., Buhler, M., Klein, O., Ende, G., et al. (2010). Initial, habitual and compulsive alcohol use is characterized by a shift of cue processing from ventral to dorsal striatum. Addiction, 105(10), 1741-1749. https://doi.org/ 10.1111/j.1360-0443.2010.03022.x.

Wang, M., Zheng, H., Du, X., \& Dong, G. (2019). Mapping internet gaming disorder using effective connectivity: A spectral dynamic causal modeling study. Addictive Behaviors, 90, 62-70. https://doi.org/10.1016/j.addbeh.2018.10.019.

Weafer, J., Crane, N. A., Gorka, S. M., Phan, K. L., \& de Wit, H. (2019). Neural correlates of inhibition and reward are negatively associated. NeuroImage, 196, 188-194. https://doi.org/10. 1016/j.neuroimage.2019.04.021.

Wetherill, R. R., Squeglia, L. M., Yang, T. T., \& Tapert, S. F. (2013). A longitudinal examination of adolescent response inhibition: Neural differences before and after the initiation of heavy drinking. Psychopharmacology, 230(4), 663-671. https://doi. org/10.1007/s00213-013-3198-2.
Whelan, R., Watts, R., Orr, C. A., Althoff, R. R., Artiges, E., Banaschewski, T., et al. (2014). Neuropsychosocial profiles of current and future adolescent alcohol misusers. Nature, 512(7513), 185. https://doi.org/10.1038/nature13402.

Yarkoni, T., Poldrack, R. A., Nichols, T. E., Van Essen, D. C., \& Wager, T. D. (2011). Large-scale automated synthesis of human functional neuroimaging data. Nature Methods, 8(8), 665-695. https://doi.org/10.1038/nmeth.1635.

Zheng, H., Hu, Y., Wang, Z., Wang, M., Du, X., \& Dong, G. (2019). Meta-analyses of the functional neural alterations in subjects with Internet gaming disorder: Similarities and differences across different paradigms. Progress in Neuro-Psychopharmacology \& Biological Psychiatry, 94, 109656-109656. https://doi. org/10.1016/j.pnpbp.2019.109656.

Zhou, F., Zimmermann, K., Xin, F., Scheele, D., Dau, W., Banger, M., et al. (2018). Shifted balance of dorsal versus ventral striatal communication with frontal reward and regulatory regions in cannabis-dependent males. Human Brain Mapping, 39(12), 5062-5073. https://doi.org/10.1002/hbm.24345.

Zilverstand, A., Huang, A. S., Alia-Klein, N., \& Goldstein, R. Z. (2018). Neuroimaging impaired response inhibition and salience attribution in human drug addiction: A systematic review. Neuron, 98(5), 886-903. https://doi.org/10.1016/j.neuron.2018.03.048. 